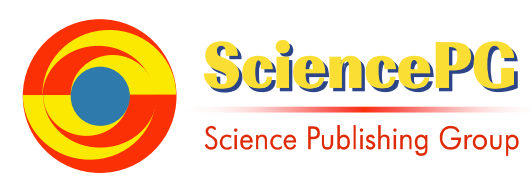

\title{
Music/multimedia technology: Melody synthesis and rhythm creation processes of the hybridized interactive algorithmic composition model
}

\author{
E. J. Garba*, G. M. Wajiga \\ Department of Computer Science, Modibbo Adama (Federal) University of Technology P.M.B. 2076 Yola, Adamawa State, Nigeria
}

Email address:

e.j.garba@mautech.edu.ng (E. J. Garba)

\section{To cite this article:}

E. J. Garba, G. M. Wajiga. Music/Multimedia Technology: Melody Synthesis and Rhythm Creation Processes of the Hybridized Interactive Algorithmic Composition Model. American Journal of Software Engineering and Applications. Vol. 3, No. 6, 2014, pp. $106-111$. doi: 10.11648/j.ajsea.20140306.17

\begin{abstract}
Music composition, by machine, requires the solution of a number of difficult problems in the fields of algorithm design, data representation, human interface design, and software engineering in general. These aforementioned problems led to the emergence of the objectives of this research. Consequently, a concept formulation was derived from the existing algorithmic composition models - where their strengths were harnessed and their weaknesses transparently subdued. This brought about the hybridization of the existing models that gave birth to Hybridized Interactive Algorithmic Composition model that leverages on the speed and accuracy of the computer to complement human creativity in music improvisation and composition. This paper presents both the melody synthesis and rhythm creation processes of the Hybridized Interactive Algorithmic Composition Model.
\end{abstract}

Keywords: Hybridized Interactive Algorithmic Composition Model, Algorithmic Composition, Melody Synthesis, Rhythm Creation

\section{Introduction}

Music is the skillful arrangement and alternation of sound and silence within a given period of time with appreciable aural perception. When musicians play instruments, sound is produced (as the sound-producing parts of the instruments vibrate/oscillate). These vibrations cause air molecules to displace one another in a systematic continuous flow, which moves away from its source. In the end, our ears perceive these air pressure fluctuations as sounds that are translated by our brains as music [1].

Musically speaking, the sounds produced are musical notes and the silences/pauses between the notes are called rests. The musical notes and rests vary in duration. That is, a note could be played for a long or short time. Thus, in contemporary music, there are six types of notes - whole, half, quarter, eighth, sixteenth and thirty-second. For proper interpretation of notes/rests duration, a piece of music is divided into portions called measures (bars). Each measure is bounded by two bars - within which a fixed number of notes/rests are required [1].

To determine the number of notes/rests in a measure, a time signature (also known as meter) is required. This is usually placed in front of a musical staff. The time signature is a fractional number written in front of any musical piece. The numerator indicates the number of beats in a measure, while the denominator specifies the type of note/rest assigned to one beat. There are so many types of meters, but the common [C] time signature is $4 / 4$ (i.e. four beats in a measure, and each beat is a quarter note) [1]. See Table 1 for details.

Table 1. Musical Notes/Rests Interpretation.

\begin{tabular}{|c|c|c|c|}
\hline Note Type & Note symbol & Rest symbol & Number of Notes/Rests \\
\hline Whole & 4 & 드 & 16 \\
\hline Half & 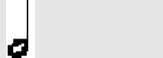 & $\overline{\underline{2}}$ & 8 \\
\hline Quarter & & है & 4 \\
\hline Eight & & $y$ & 2 \\
\hline Sixteenth & & y & 1. \\
\hline
\end{tabular}




\section{Computability of Music}

According to [1], music is the alternation of sound and silence. It is therefore obvious that the concept of music conforms to binary theory; where only the values of 0 and 1 are considered. The binary value of " 0 " indicates certainly that an event will not occur; while " 1 " indicates certainly the event will occur.

Logically put, when sound is produced (or a note/tone is played) the binary value is 1 , otherwise it is 0 . That is:

- $\quad 1 \rightarrow$ sound is produced (or a note/tone is played)

- $\quad 0 \rightarrow$ silence (no note is played)

Therefore, the binary musical concept perceives music as a stream of $1 \mathrm{~s}$ and $0 \mathrm{~s}$. This therefore, means that musical ideas and concepts are logical - hence computable.

\section{Algorithmic Composition}

Algorithmic composition is the technique of using algorithms to create music. Music composition is usually a complex and difficult task for the people not having musical knowledge or skill, and composer's expertise plays an important role. Nevertheless, there have been many studies on automatic music composition using computer since the conception of the computer, and some automatic music composition models have been proposed [2].

The formal technique of generating music was adopted again in the 20th century. The tone-row technique was introduced by Arnold Schönberg at the beginning of the century and further developed into serialism by Anton Webern and his successors [3]. In the serial technique music was factorized into parameters such as pitch, duration, and timbre that were controlled separately. A permutation was chosen from the possible values of each parameter and arranged into a row. The parameter values changed according to the row or its inversions or retrogrades [4].

According to [3], mentions some common techniques of algorithmic composition; which include state machines, rulebased, grammars, stochastic processes, and genetic algorithms. Therefore, Systems for the algorithmic composition of music can be conveniently categorized into three types; rule-based systems, systems which learn by example - knowledge-based and genetic algorithms [5] and $[6]$.

\section{Concept Formulation}

A complete theory of general creativity, or even musical creativity, remains elusive. While many attempts have been made to study and document the creative process [7],[8],[9],[10] for example, any generality seems difficult to uncover. In many cases people do not know how or why they make creative decisions, and much of the creative process is difficult to repeat in controlled experiments. The question of whether creativity is computable is an issue of even greater controversy. Many researchers have argued against a computable model of creativity because, simply put, the underlying mental processes are not computable. These arguments depend largely on speculation and the personal opinion of the authors.

For instance, it has been observed that almost all forms of music involve repetition [11], either of individual sequences of notes or at some higher levels of structural grouping. Often these repetitions appear at a number of different levels simultaneously. Some compositions repeat patterns that are slightly changed at each repetition, or revolve around some musical 'theme', whereby complex harmonic, timing or key shifts in a basic theme provide a pleasing musical diversity.

It is also commonly known that what allows us to identify an individual piece of music is the change in pitch between notes, not the pitch of the notes themselves. We can change the key of a composition (equivalent to multiplying all frequencies by some fixed amount) and still recognise the melody [12].

According to the generative theory of tonal music, music is built from an inventory of notes and a set of rules. The rules assemble notes into a sequence and organize them into hierarchical structures of music cognition. To understand a piece means to assemble these mental structures as we listen to the piece [13].

A hybridized interactive algorithmic composition model was developed from the existing algorithmic composition models in order to minimize the weaknesses experienced when such models are used singlehandedly in music composition. The hybridization of the models (at different stages of composition) capitalized on the advantages of such models. The models for algorithmic composition used included: Mathematical [14], Grammar (Rule-Based) [15], stochastic [16], Knowledge-based [3], and Evolutionary (Genetic Algorithm) [17].

\subsection{Rhythm Creation Process}

Rhythmic composition is based on both mathematical and grammar models [18]. Therefore, in any contemporary music composition, the most important parameter is the meter indicated as a time signature on the musical staff.

That is,

$$
\text { met }=\frac{m}{n}
$$

Where: met stands for meter, $m$ is the numerator that indicates the number of beats in a measure, $\mathrm{n}$ is the denominator that specifies the type of note/rest assigned to one beat. This means, therefore that a beat in any rhythm could be represented with streams of binary digits (bits) known as Binary Code (BC). See Table 2 for details: 
Table 2. Beat Binary Code.

\begin{tabular}{|c|c|c|c|c|c|}
\hline \multirow{2}{*}{ Note Type } & \multirow{2}{*}{ Note symbol } & \multirow{2}{*}{ Rest symbol } & \multicolumn{3}{|c|}{ Beat Binary Code (this is the stream of bits required to represent a beat) } \\
\hline & & & Bits & Note Binary Value & Rest Binary Value \\
\hline Whole & $\infty$ & $=$ & 16 & 1000000000000000 & 0000000000000000 \\
\hline Half & $\Rightarrow$ & $=$ & 8 & 10000000 & 00000000 \\
\hline Quarter & & है & 4 & 1000 & 0000 \\
\hline Eight & & $y$ & 2 & 10 & 00 \\
\hline Sixteenth & & धy & 1 & 1 & 0 \\
\hline
\end{tabular}

Therefore,

$$
B_{B C}=\operatorname{Random}\left(\operatorname{Abs}\left(2^{n}-1\right)\right)
$$

Where: B stands for Beat, BC stands for Binary Code, Abs stands for absolute value and $\mathrm{n}$ is the denominator that specifies the type of note/rest assigned to one beat in a meter.

In contemporary music, the bar/measure forms the basic rhythmic and melodic unit. That means that Rhythm (R) Binary Code, which is made up of a measure, is a function of the given meter. That is:

$$
R_{B C}=f(m e t)
$$

$$
R_{B C}=\left\{B_{B C_{1}}, B_{B C_{2}}, \ldots, B_{B C_{m}}\right\}
$$

Where: R stands for Rhythm, BC stands for Binary Code and $\mathrm{m}$ is the numerator that indicates the number of beats in a measure of a given meter. Note: $\mathrm{R}_{\mathrm{BC}}$ logically represents a musical rhythmic pattern (RP). Therefore, $R P=R_{B C}$.

For example, if the meter (of a given piece of music) is $3 / 4$, then it means there will be only three beats (or three Beat Binary Code) and each of the beats is assigned a quarter notes (4 bits); thus, the following mathematical, logical/binary and musical expressions are obtainable in

\begin{tabular}{|c|c|c|}
\hline Mathematical expression & Logical/Binary expression & Musical expression \\
\hline $\begin{array}{l}\text { Based on equation ( } 4) \text {, the following denary } \\
\text { values could be randomly or interactively } \\
\text { generated: } R P=\{10,8,14\} \\
\text { This equation has only three beat values because } \\
\mathrm{m}=3 \text { from the given meter of } 3 / 4 \text {. }\end{array}$ & $\begin{array}{l}\mathrm{R}_{\mathrm{BC}}=\{1010,1000,1110\} \\
\text { This is the binary/logical equivalent of the } \\
\text { denary values of the RP. Each Beat Binary } \\
\text { Code is allocated } 4 \text { bits; } \mathrm{n}=4 \text { (a quarter } \\
\text { note) from the meter of } 3 / 4 \text {. }\end{array}$ & $\begin{array}{l}\text { From Table2, it should be noted that } 1 \text { bit represents a } \\
\text { sixteenth note. Therefore, the musical expression of } \\
\text { this RP is: }\end{array}$ \\
\hline
\end{tabular}
Table 3 .

Table 3. Example of Mathematical, logical/binary and musical expressions.

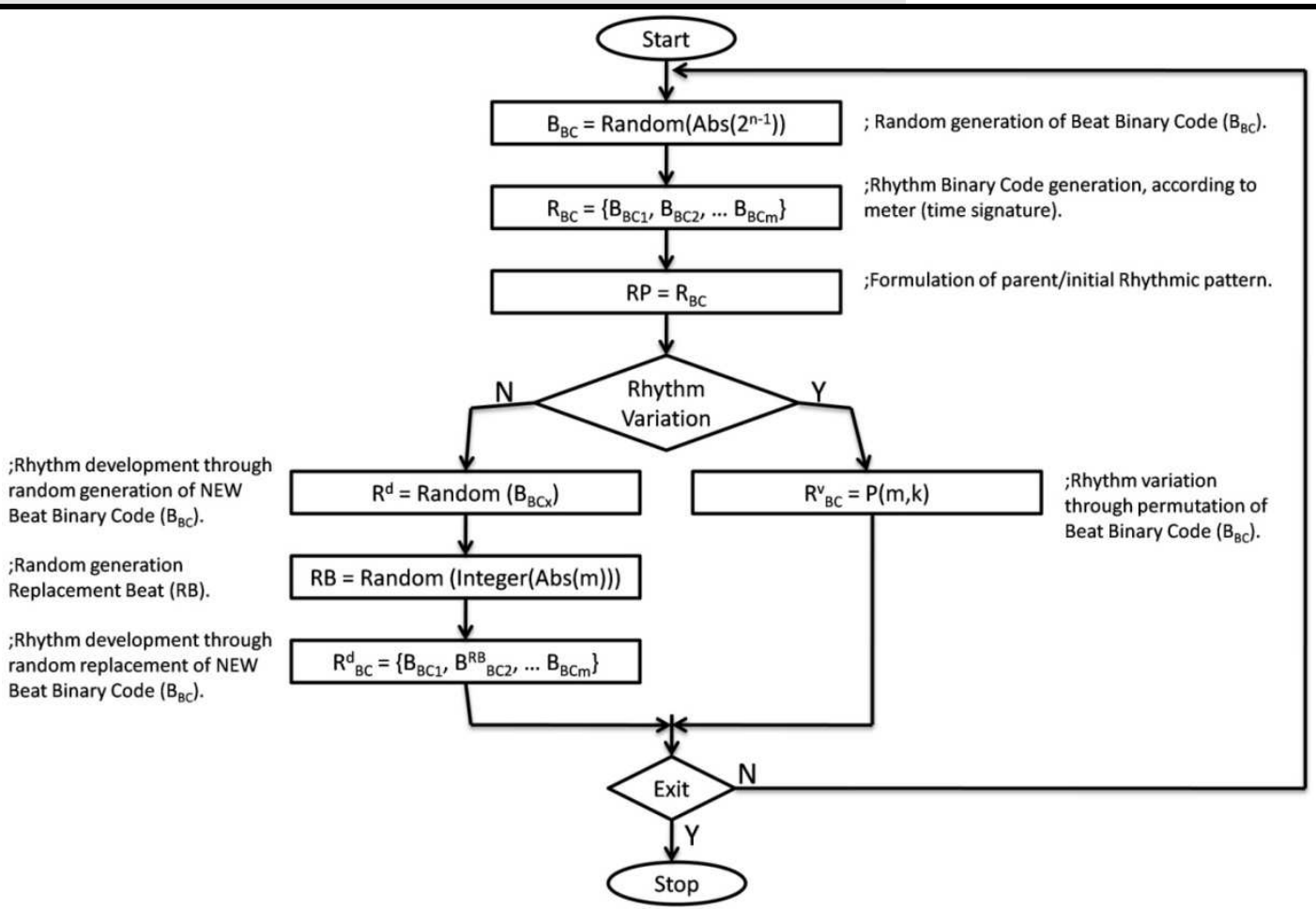

Figure 1. Rhythm Creation Process[19]. 
The rhythm creation process of the Hybridized Interactive Algorithmic Composition Model is developed from the concept formulation of this research [19]. Here, the beat binary code, which could be generated through the process of randomization, makes up a rhythm binary code (which in essence is a rhythmic pattern based on the given meter/time signature).

Variations of the rhythmic patterns could be generated or created through randomization or permutation of the beat binary code. The result of the rhythm creation process of the Hybridized Interactive Algorithmic Composition Model is a rhythmic pattern devoid of melody.

\subsection{Melody Creation Process}

According to [20], the key problem is to get explicit musical knowledge, which is both formally represented and computationally useful. With this knowledge, the composition system is built using programming techniques. The composition of music was discussed at two levels. On the level of microstructure, relations between notes in horizontal direction (melodic intervals) and in vertical (synchronous) direction (harmony) were discussed. Macrostructure deals with the larger structural parts in horizontal direction, for instance, the length of phrases and their thematic and tonal relations. The vertical (synchronous) direction is described by harmony. Voice leading rules (for instance, the forbidden parallel fifth)-rule combine the vertical and micro structurally horizontal direction. The processes of classical music composition were divided into several subtasks: Using a top-down-approach, it started with planning the macrostructure of the piece.

\subsection{Compositional Parameters and Operational Constraints}

Some of the key parameters for music composition include scale/key, meter (time signature), tempo, articulations and dynamics. Beat melody/motif is a melodic sequence that consists of notes in one beat. Beat motif is determined by the composer [21].

$$
\text { Beat Motif }=f(\text { beat note in a bar })
$$

$$
\text { Beat Motif }=f\left(\text { beat note in terms of } 16^{\text {th }} \text { note }\right)
$$

\subsubsection{Operational Note Type}

The operational note type is the $16^{\text {th }}$ note. This means that the Beat Motif is made of $16^{\text {th }}$ notes of a beat. The number of $16^{\text {th }}$ notes in the Beat Motif depends on the value of $n$ from the given meter. For instance, if a meter $(\mathrm{m} / \mathrm{n})$ is $6 / 8$, it means there are six $8^{\text {th }}$ notes in the bar. Note that $\mathrm{n}=8$ - meaning each beat is an $8^{\text {th }}$; and because the Beat Motif is expressed in terms of $16^{\text {th }}$ notes, then the Beat Motif is made up of two $16^{\text {th }}$ notes.

\subsubsection{Octave Range}

This determines the types of pitches to be used in the musical composition. Here, the pitches were denoted using the Pitch-Octave convention. In this convention, alphabets are used to denote pitch classes while the number indicates the octave. For instance, C5 means pitch class " $\mathrm{C}$ " and octave "5". Therefore, for instance, an octave range of $\mathrm{C} 3$ B5 means that during composition, notes/pitches that fall within this range are eligible for usage.

\subsubsection{Operational Domains}

It determines the possible pitches that could be assigned to one note in the Beat Motif. Therefore, the operational domains of a note is $\{\mathrm{P} 1, \mathrm{P} 2, \ldots, \mathrm{Pmax}\}$. Where: $\mathrm{P}$ stands for pitch and max is the total number of pitches as specified by the Octave Range. For instance, if the number of notes in the Beat Motif is $\mathrm{U}$, the operational domain is $[\{\mathrm{P} 1, \mathrm{P} 2, \ldots$, $P \max \} 1,\{\mathrm{P} 1, \mathrm{P} 2, \ldots, P \max \} 2, \ldots,\{\mathrm{P} 1, \mathrm{P} 2, \ldots, P \max \} \mathrm{U}]$. Therefore, the total possible Beat Motif from such an operation domain is $\left(\sum_{i=1}^{\max } P\right)^{U}$.

\subsubsection{PC Segment}

The Pitch Class (PC) segment consists of a bar of melodic sequence. Note that the melodic sequence is made up of Beat Motifs (note that the number of Beat Motifs corresponds to the number of beats in a bar). That is:

$$
\mathrm{PC}_{\mathrm{seg}}=\left\{\mathrm{BM}_{1}, \mathrm{BM}_{2}, \ldots, \mathrm{BM}_{\mathrm{m}}\right\}
$$

Where: $\mathrm{PC}_{\text {seg }}$ stands for PC segment, BM stands for Beat Motif and $\mathrm{m}$ stands for number of beats in a bar from a given meter.

The PC segment is defined either by the composer or automatically generated by the computer. The user could define all the BMs which make up the PC segment; alternatively, the user could define the initial BM while the rest of the BMs are automatically generated by randomization or permutation of the notes of the initial BM.

For example, if given a $4 / 4$ meter, a beat motif of four $16^{\text {th }}$ notes of $\mathrm{c}, \mathrm{e}, \mathrm{g}$ and $\mathrm{a}$; then $\mathrm{BM}=(\mathrm{c}, \mathrm{e}, \mathrm{g}, \mathrm{a})$. This means that the pitch class values of the $\mathrm{BM}=(1,5,8,10)$. However, through randomization and permutation, there are 23 more possible beat motifs. From the possible 24 beat motifs, just four BMs were required (according to the meter $-4 / 4$ ) to form the PC segment. This gives rise to 255,024 possible PC segments. One of PC segments could be this:

$$
\mathrm{PC}_{\mathrm{seg}}=\{(\mathrm{c}, \mathrm{e}, \mathrm{g}, \mathrm{a}),(\mathrm{a}, \mathrm{g}, \mathrm{e}, \mathrm{c}),(\mathrm{c}, \mathrm{c}, \mathrm{c}, \mathrm{c}),(\mathrm{e}, \mathrm{g}, \mathrm{c}, \mathrm{a})\}
$$

\section{Prime Form}

The Prime Form is created when rhythm is now added to the PC segment. Note that the PC segment contains melodic sequence dependent on the value of beat in the given meter. Therefore, Prime Form $=$ PC Segment + Rhythm.

For instance, given this PC segment:

$$
\mathrm{PC}_{\mathrm{seg}}=\{(\mathrm{c}, \mathrm{e}, \mathrm{g}, \mathrm{a}),(\mathrm{a}, \mathrm{g}, \mathrm{e}, \mathrm{c}),(\mathrm{c}, \mathrm{c}, \mathrm{c}, \mathrm{c}),(\mathrm{e}, \mathrm{g}, \mathrm{c}, \mathrm{a})\}
$$

While the rhythm pattern is:

$$
\mathrm{R}_{\mathrm{BC}}=\{(1010),(1110),(1000),(1101)\}
$$


In order to get a prime form from the PC segment and rhythm pattern, the only notes picked were those where the

rhythm binary code value is 1 . See Table 4 .

Table 4. Prime Form Creation.

\begin{tabular}{lllllllllllllllll}
\hline Note & $\mathbf{1}$ & $\mathbf{2}$ & $\mathbf{3}$ & $\mathbf{4}$ & $\mathbf{5}$ & $\mathbf{6}$ & $\mathbf{7}$ & $\mathbf{8}$ & $\mathbf{9}$ & $\mathbf{1 0}$ & $\mathbf{1 1}$ & $\mathbf{1 2}$ & $\mathbf{1 3}$ & $\mathbf{1 4}$ & $\mathbf{1 5}$ & $\mathbf{1 6}$ \\
\hline $\mathrm{PC}_{\mathrm{seg}}$ & $\mathrm{c}$ & $\mathrm{e}$ & $\mathrm{g}$ & $\mathrm{a}$ & $\mathrm{a}$ & $\mathrm{g}$ & $\mathrm{e}$ & $\mathrm{c}$ & $\mathrm{c}$ & $\mathrm{c}$ & $\mathrm{c}$ & $\mathrm{c}$ & $\mathrm{e}$ & $\mathrm{g}$ & $\mathrm{c}$ & $\mathrm{a}$ \\
$\mathrm{R}_{\mathrm{BC}}$ & 1 & 0 & 1 & 0 & 1 & 1 & 1 & 0 & 1 & 0 & 0 & 0 & 1 & 1 & 0 \\
Prime Form & $\mathrm{c}$ & & $\mathrm{g}$ & & $\mathrm{a}$ & $\mathrm{g}$ & $\mathrm{e}$ & & $\mathrm{c}$ & & & & $\mathrm{e}$ & $\mathrm{g}$ & & $\mathrm{a}$ \\
\hline
\end{tabular}

\section{Note Duration Parser}

The note duration parser is used for refining the prime form where the " " symbol was appended to a note preceding it or the "-" symbol is used as rest. Note duration is determined through a stochastic process of probability and randomization. The end results of this stage were prime forms with different combinations of note duration.

From prime form: Prime Form: $\left\{\left(\mathrm{c}, \ldots, \mathrm{g}, \_\right),\left(\mathrm{a}, \mathrm{g}, \mathrm{e}, \_\right),(\mathrm{c}\right.$, _, , _ $),(\mathrm{e}, \mathrm{g}, \ldots, \mathrm{a})\}$, the following results are possible:

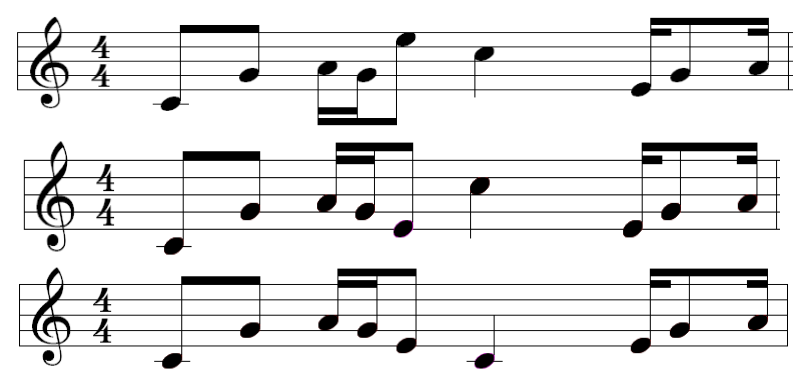

Note that with just one PC segment, several prime forms could be created from different rhythm variations. That is, one PC segment could be applied to several rhythm patterns; likewise, one rhythm pattern could be applied to several PC segments. This is the flexibility of Hybridized Interactive Algorithmic Composition (HIAC) model.

\section{Composition Rules Parser and Optimizer}

At this point, the Composition Rules Parser analyzes the Prime Form based on the basic prevalent contemporary music composition rules; after which, the Prime Form was further refined by the Optimizer. The optimization process could be done either manually by the composer or automatically. The final result of this stage is known as Optimized Prime Form. See the following example of an Optimized Prime Form:

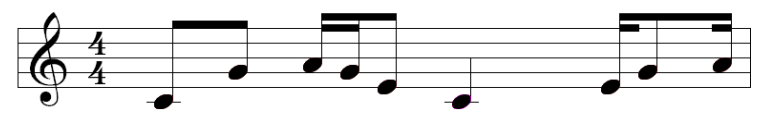

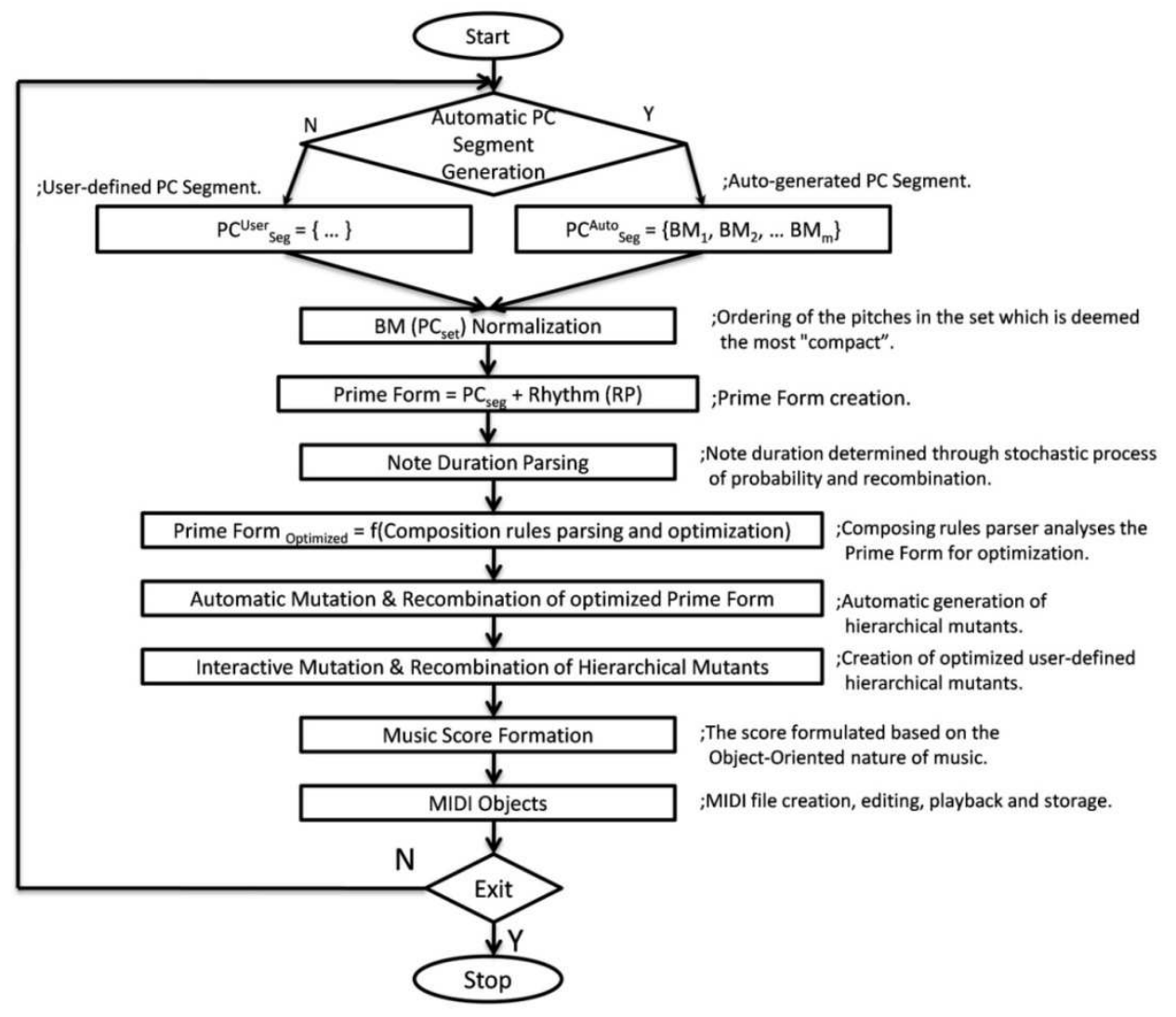

Figure 2. Melody Creation Process[19]. 


\section{Conclusion}

The above formulated melody synthesis and rhythm creation processes of the Hybridized Interactive Algorithmic Composition Model have their place in music composition; thereby, offsetting the complex task of music creation from the human composer to the machine via algorithms. This ensures speed and accuracy with minimal and interactive interference by the human composer.

\section{References}

[1] Garba, E. J. (2003). Computer Music - Rhythm Programming, Processing and Mastering. Trafford Publishing, Canada.

[2] Unehara, M. and T. Onisawa. (2009). Construction of Music Composition System with Interactive Genetic Algorithm. University of Tsukuba, 1-1-1 Tennodai, Tsukuba, Ibaraki, 3058573 , JAPAN. http://www.idemployee.id.tue.nl/g.w.m.rauterberg/conferences /CD_doNotOpen/ADC/final_paper/549.pdf.

[3] Järveläinen, H. (2000). Algorithmic Musical Composition. Helsinki University of Technology, Telecommunications software and multimedia laboratory. Retrieved December 5, 2007, from www.tml.tkk.fi/Studies/Tik111.080/2000/papers/hanna/alco.pdf.

[4] Grout, D. J. (1996). History of Western Music. W.W. Norton \& Company, 5 th edition.

[5] Todd, P. M. and G. M. Werner. (1999). Frankensteinian Methods for Evolutionary Music Composition. In Griffith and Todd, P. M. (Eds.) Musical networks: Parallel perception and performance, 313-339.

[6] Espi, D., P. J. Ponce de Leon, C. Perez-Sancho, D. Rizo, J. M. Inesta, F. Moreno-Seco, and A. Pertusa. (2009). A Cooperative Approach to Style-Oriented Music Composition. Departamento de Lenguajes y Sistemas Informaticos University of Alicante, Spain. http://193.145.231.49/repositori/grfia/pubs/186/wijcai07.pdf.

[7] Barron, F. (1969). Creative Person and Creative Process. Holt, Rinehart and Winston, Inc.

[8] Crutchfield, R.S. (1973). The Creative Process. In Creativity: Theory and Research. College and University Press, Bloomberg, M., $54-74$.

[9] Schillinger, J. (1948). The Mathematical Basis of the Arts. The Philosophical Library, New York.

[10] Johnson-Laird, P.N. (1993). Human and Machine Thinking. Lawerance Eribaum Associates.
[11] Leach, J. and Fitch, J. (1995). Nature, Music, and Algorithmic Composition. Computer Music Journal 19(2), 23 - 33.

[12] McCormack, J. (2009). Grammar Based Music Composition. Computer Science Department Monash University, Clayton Victoria 3168 . http://www.csse.monash.edu.au/ jonmc/research/Papers/LsystemsMusic.pdf.

[13] de Mantaras, R. L. and J. L. Arcos. (2002). AI and Music: From Composition to Expressive Performance. American Association for Artificial Intelligence.

[14] Gilkerson, J., Li, W. and Owen, D. (2005). An Introduction to Random Number Generators and Monte Carlo Methods. Retrieved June 1, 2007, from http://www.mgnet.org/ douglas/Classes/cs521/rng$\mathrm{mc} /$ RandomMonteCarlo2005.ppt.

[15] Towsey, M., Brown, A., Wright, S. and Diederich, J. (2009). Towards Melodic Extension Using Genetic Algorithms. Queensland University of Technology Kelvin Grove, QLD 4059, Australia. Retrieved September 23, 2009, fromhttp://eprints.qut.edu.au/169/1/towsey.pdf.

[16] Microsoft Encarta Encyclopedia. (2009). Stochastic. Microsoft Encarta 2009 [DVD]. Redmond, WA, USA: Microsoft Corporation.

[17] Holland, J.H. (1975). Adaptation in Natural and Artificial Systems. USA: Ann Arbor, University of Michigan Press (Second edition: MIT Press, 1992).

[18] Garba, E. J. and Wajiga, G. M. (2011). A Review of Multimedia and Music Technology: Mathematical Foundations of Rhythm Generations in Algorithmic Composition, Bagale Journal of Pure and Applied Sciences, Volume 8, Number 1, December 2011, pp. 64-74. http://www.mautech.edu.ng/

[19] Garba, E. J. (2012). Multimedia Technology: A Software Framework for Interactive Music Composition. ( $\mathrm{PhD}$ Thesis in Music/Multimedia Technology, School of Pure and Applied Sciences Federal University of Technology, Yola, Nigeria).

[20] Lothe, M. (2007). Knowledge-based Composition of Classical Minuets by a Computer. Universitat Stuttgart; Institut fur Informatik Breitwiesenstr. 20-22 D-70565 Stuttgart, Germany. Retrieved August 10, 2008, from www.informatik.unistuttgart.de/ifi/is/Forschung/Papiere/mathis/aisb2000-loethepn.ps.

[21] Garba, E. J., Wajiga, G. M. and Oye, N. D. (2011). Multimedia and Music Technology: Mathematical Foundations of Melody Synthesis in Algorithmic Composition, International Journal of Computer Vision \& Applications, Volume 1, Number 1, April 2011, pp. 9-14. http://www.3kbioxml.com/3k/index.php/IJCVA 